\title{
SCIDoC
}

International Journal of Anatomy and Applied Physiology (IJAAP)

ISSN: 2572-7451

\section{A Rare Morphological Variation of Renal artery in a Sri Lankan Cadaver}

Lanka Ranaweera $^{1 *}$, Suneth Weerasinghe ${ }^{2}$, Kasun Withana ${ }^{3}$

${ }^{1}$ Department of Anatomy, Faculty of Medicine, University of Kelaniya, Ragama, Sri Lanka.

${ }^{2}$ Teaching Hospital, Peradeniya, Sri Lanka.

${ }^{3}$ Base Hospital, Mulleriyawa, Sri Lanka.

\section{Abstract}

Arterial blood supply of kidney is carried out via a pair of renal arteries which are the direct branches of abdominal aorta. In a routine dissection of a 72 year old male cadaver at the Faculty of Medicine, University of Kelaniya, Sri Lanka a variation of two hilar arteries with one inferior polar artery of right kidney was observed. This deviation from the normal renal arterial pattern is a rare occurrences and such knowledge of variation is essential for avoiding undue vascular and urological complications at the time of donor nephrectomy and transplant recipient.

Keywords: Renal Artery; Variation; Kidney; Sri Lanka.

\section{Introduction}

Kidneys are two bean shaped organs situated retroperitonealy in the posterior abdominal region. They lie in the extra peritoneal connective tissue, lateral to the vertebral column. In the supine position, the kidneys extend from approximately vertebra T12 superiorly to vertebra L3 inferiorly. The right kidney is situated lower than the left and the left kidney is longer and slender when compared to the right. Usually each kidney is supplied by a single renal artery, a lateral branch of the abdominal aorta. The right renal artery arises just inferior to the origin of the superior mesenteric artery between vertebrae L1 and L2. It is longer and passes posterior to the inferior vena cava. When renal artery approaches the renal hilum it divides into anterior and posterior branches to supply the renal parenchyma. Any variation of the origin, number of branches and their distribution occur in the renal artery indicates the importance of the field of surgery and radiology. The current study present an interesting rare case of right renal artery variation that clinician should consider this as one of the important co-existing renal artery variations.

\section{Case Report}

During a routine dissection in the Department of Anatomy, Faculty of Medicine, University of Kelaniya, Sri Lanka, a rare variation of renal artery was found in a 72 year old male cadaver. There were three distinct arteries arising from the aorta at the level of origin of the normal renal artery on the right side. We traced these vessels carefully and found that two arteries entered the right kidney at the hilum and other one at the inferior pole (Figure 1).

the three branches the superior branch (SB) entered the kidney at the center of the hilum, and it measured $4.4 \mathrm{~cm}$ in length. The middle branch $(\mathrm{MB})$ was $5.7 \mathrm{~cm}$ long and it commenced just below and almost touching the superior branch. After a straight course to the right kidney it appeared to give off two small extra hilar branches to the upper pole ( $\mathrm{a}$ and $\mathrm{b}$ ) of the kidney following which the main trunk (c) entered the renal hilum just below the superior branch (Figure 2). The inferior branch (IB) arose from the aorta $1.7 \mathrm{~cm}$ below the middle branch, and it also had a somewhat straight course for $5.2 \mathrm{~cm}$ towards the inferior pole of the right kidney, just below the renal hilum (Figure 2).

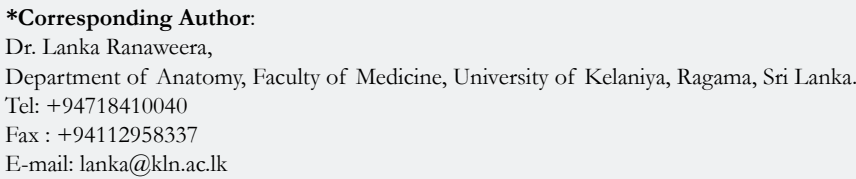

Copyright: Lanka Ranaweera ${ }^{\circ}$ 2021. This is an open-access article distributed under the terms of the Creative Commons Attribution License, which permits unrestricted use, distribution and reproduction in any medium, provided the original author and source are credited. 
On the left side there was one renal artery arising from the abdominal aorta approximately at the same level of origin of the $\mathrm{SB}$ and $\mathrm{MB}$ of the right renal artery and entering at the hilum of the left kidney.

\section{Discussion}

Embryological basis of renal artery variation is reflected by the manner in which the blood supply continually changes during embryonic and early fetal life. As the kidneys "ascend" from the pelvis, they receive their blood supply from the vessels close to them. Initially the renal arteries are branches of the common iliac arteries, With the ascent of the kidneys they receive the blood supply from the distal end of the aorta up to the abdominal aorta until the kidney comes into contact with the suprarenal gland in the ninth week; their "ascent" stops. These most cranial branches become the permanent renal arteries and normally caudal branches undergo involution and disappear. Accessory (supernumerary) renal arteries usually arise from the aorta superior or inferior to the main renal artery and follow it to the hilum, although some may enter the kidneys directly, at the superior or inferior poles [1]. We observed two renal arteries entering the hilum and one accessory branch entering the inferior pole of the right kidney in our case.

Variations of the renal arteries have been well documented in literature by angiographic and anatomical studies [2-6]. Multiplicity of renal arteries is found to be a common variation and its prevalence varies from $20 \%$ to $50 \%[3,7,8]$. Sampaio and Passos had analyszed 266 kidneys and they reported 12 types of renal artery variations and according to them, our case ( 2 hilar arteries with 1 inferior polar artery) is a rare variant that has a prevalence of $0.7 \%$. More common variations are 1 hilar artery in $53.3 \%$ of cases, 1 hilar artery with 1 superior pole extra-hilar branch in 14.3\%, 2 hilar arteries in $7.9 \%, 3$ hilar arteries in $1.9 \%$, superior polar artery in $6.8 \%$, and inferior polar artery in 5.3\% [8].

Khamanarong and colleagues had dissected a total of 267 Thai cadavers. Their anatomical findings included: a single hilar artery in $82 \%$ of cases, double renal arteries in $17 \%$ of cases (one hilar artery with an upper polar artery in 7\%, two hilar arteries in 7\% and one hilar artery combined with one lower polar artery in 3\%) and triple renal arteries occurred in 1\% (two hilar arteries with one upper polar artery in $0.4 \%$ and two hilar arteries with one lower polar artery in $0.6 \%$ ) [5].

Hemanth and colleagues observed additional arteries in 52 $(28.2 \%)$ out of a total of 184 kidneys; 34 kidneys had one artery $(18.5 \%)$ and 18 kidneys had two additional arteries (9.7\%). The frequency of one additional artery was $32.3 \%$ on the right side and $67.7 \%$ on the left side. The frequency of two additional arteries was $50 \%$ on the right side and $50 \%$ on the left side [6].

The anatomical vascular variation in our case study (two hilar arteries with one inferior polar artery) is very important because of its rare occurrence which has been recorded as having a prevalence of $0.6 \%$ in the literature [5].

Knowledge of these renal artery variations play a crucial role in urological surgeries as surgical hazards are likely to occur during renal transplantations and renal surgeries, which may lead to various clinical conditions like hydroneprosis and hypertension.

The incidence of donor kidney vascular anomalies ranges from $18 \%$ to $30 \%$ [9]. Coen LD and Raftery AT observed that multiple renal arteries occurred bilaterally in $10.2 \%$ and unilaterally in $20.8 \%$ of donors with a total incidence of $31 \%$ in relation to 513 kidney transplants [10]. Most of the research emphasized that there was a higher incidence of vascular-related complications

Figure 1. Anterior view of the right kidney. Ao - Abdominal Aorta, U - Ureter, SB - Superior branch renal artery, MB - Middle branch renal artery, IB - Inferior branch renal artery, RK - Right Kidney.

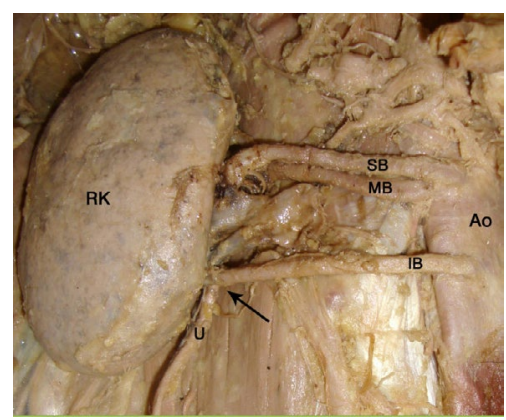

Figure 2. Posterior view of the right kidney. Ao - Abdominal Aorta, U - Ureter, SB - Superior branch renal artery, MB - Middle branch renal artery, IB - Inferior branch renal artery, RK - Right Kidney, a - first extra hilar branch of MB to upper pole, b - second extra hilar branch of MB to upper pole, c - Main trunk of MB to renal hilum, RV - Renal vein.

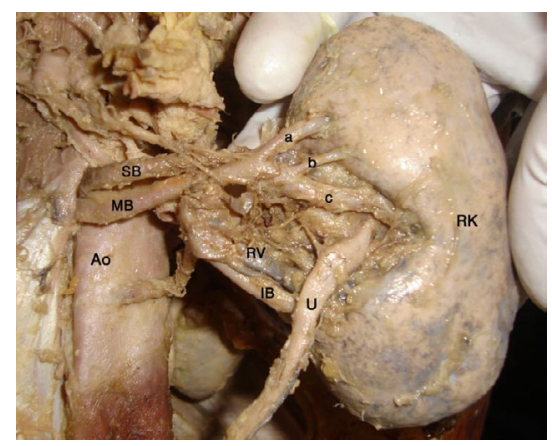


following transplantation of kidneys with multiple renal arteries [8]. Since these vessels are end arteries, damage to them will lead to segmental ischemia with subsequent hypertension.

A non-surgical complication is the presence of an accessory vessel to the inferior pole of the kidney, crossing anterior to the ureter and there by obstructing the ureter causing hyronephrosis [5]. We found a similar pattern in our case study as indicated by the black arrow in the Figure 1.

\section{Conclusion}

The two hilar arteries with one inferior polar artery of right kidney is a very rare condition and it should be considered as one of the renal vessels variations at the time of donor nephrectomy and recipient transplant, in order to prevent vascular and urological complications.

\section{Acknowledgements and Declarations}

We dedicate this article to the memory of people who donated their bodies to the Department of Anatomy, Faculty of Medicine, University of Kelaniya, Ragama, Sri Lanka, through body donation program. There is no conflict of interest to declare.

\section{References}

[1]. Wozniak WT. Origin of the renal arteries from sides of aorta. Folia Morphol (Warsz). 2000;58(4):259-61. Pubmed PMID: 11000882.

[2]. Jeffery RF. Unusual origins of renal arteries. Radiology. 1972 Feb;102(2):30910. Pubmed PMID: 5009930.

[3]. Awojobi OA, Ogunbiyi OA, Nkposong EO. Unusual relationship of multiple renal arteries. Urology. 1983 Feb;21(2):205-6. Pubmed PMID: 6823735.

[4]. Dalçik C, Colak T, Ozbek A, Dalçik H. Unusual origin of the right renal artery: a case report. Surg Radiol Anat. 2000;22(2):117-8. Pubmed PMID: 10959679.

[5]. Khamanarong K, Prachaney P, Utraravichien A, Tong-Un T, Sripaoraya K. Anatomy of renal arterial supply. Clin Anat. 2004 May;17(4):334-6. Pubmed PMID: 15108340.

[6]. Hemanth K, Sree LD, Jothi SS, Rajeswararao N, Sujatha N. Presence of renal artery variation and its surgical correlation. Int $\mathrm{J}$ of Medi and Clin Res 2012;3:176-9

[7]. ANSON BJ, DASELER EH. Common variations in renal anatomy, affecting blood supply, form, and topography. Surg Gynecol Obstet. 1961 Apr;112:439-49. Pubmed PMID: 13683701.

[8]. Sampaio FJ, Passos MA. Renal arteries: anatomic study for surgical and radiological practice. Surg Radiol Anat. 1992;14(2):113-7. Pubmed PMID: 1641734 .

[9]. De Coppi P, Giuliani S, Fusaro F, Zanatta C, Zacchello G, Gamba P, et al. Cadaver kidney transplantation and vascular anomalies: a pediatric experience. Transplantation. 2006 Oct 27;82(8):1042-5. Pubmed PMID: 17060852.

[10]. Coen LD, Raftery AT. Anatomical variations of the renal arteries and renal transplantation. Clin Anat 2005;5:425-32. 